\title{
Hallazgos histopatológicos en sangrado uterino anormal en pacientes posmenopaúsicas del Hospital de Clínicas
}

\author{
Histopathological findings in abnormal uterine bleeding in postmenopausal \\ patients from Hospital de Clínicas \\ Jorge Gavilán $\mathbb{B}^{1}$, Hugo Arellano
}

${ }^{1}$ Universidad Nacional de Asunción, Facultad de Ciencias Médicas, San Lorenzo, Paraguay.



Recibido: 01/02/2021

Revisado: 24/03/2021

Aceptado:30/04/2021

\section{Autor correspondiente}

\section{Jorge Gavilán}

Universidad Nacional de Asunción,

San Lorenzo, Paraguay

yoryigavilanh@gmail.com

\section{Conflictos de interés}

Los autores declaran no poseer conflictos de interés.

\section{Fuente de financiación}

Los autores no recibieron apoyo financiero para la investigación, autoría y/o publicación de este artículo.

Este artículo es publicado bajo una licencia de Creative Commons Reconocimiento 4.0 Internacional.



\section{RESUMEN}

Introducción: El sangrado uterino anormal en la posmenopausia es cualquier sangrado uterino que se presenta después de un año de amenorrea en la mujer en edad postmenopáusica, sin uso de terapia hormonal. Metodología: Es un estudio observacional, descriptivo y temporalmente retrospectivo. El muestreo es no probabilístico de casos consecutivos. La población accesible son pacientes posmenopáusicas con sangrado uterino anormal que fueron sometidas a biopsia de endometrio en la cátedra de Ginecología y Obstetricia del Hospital de Clínicas de la Universidad Nacional de Asunción en el período enero 2019 a diciembre 2020. Resultados: Sobre el hallazgo histopatológico se puede ver que el 30,8 \% fue pólipo endometrial, el 24,2 \% mucosa endometrial normal, el $11 \%$ adenocarcinoma. Discusión: El sangrado uterino anormal ya sido investigado en el Hospital de Clínicas por Franco-Domínguez quien llega a la conclusión de que la prevalencia de hemorragia uterina anormal de causa orgánica es $16,3 \%$. El principal motivo de consulta es la hipermenorrea (63,3\%). Los diagnósticos finales fueron mioma uterino (57 \%) y las patologías endometriales. La mayoría fue sometida a cirugía (71 \%) y $38 \%$ presentó anemia

\section{ABSTRACT}

Introduction: Abnormal uterine bleeding in postmenopause is any uterine bleeding that occurs after one year of amenorrhea in women of postmenopausal age, without the use of hormonal therapy. Methods: It is an observational, descriptive and temporally retrospective study. The sampling is non-probabilistic of consecutive cases. The accessible population are postmenopausal patients with abnormal uterine bleeding who underwent endometrial biopsy in the Department of Gynecology and Obstetrics of the Hospital de Clínicas of the National University of Asunción in the period January 2019 to December 2020. Results: About the histopathological finding was You can see that $30.8 \%$ were endometrial polyp, $24.2 \%$ normal endometrial mucosa, $11 \%$ adenocarcinoma. Discussion: The abnormal uterine bleeding has already been investigated at the Hospital de Clínicas by Franco-Domínguez who concludes that the prevalence of abnormal uterine bleeding of organic cause is $16.3 \%$. The main reason for consultation is hypermenorrhea (63.3\%). The final diagnoses were uterine myoma (57\%) and endometrial pathologies. Most underwent surgery $(71 \%)$ and $38 \%$ had anemia.

Keywords: Amenorrhea; Biopsy; Uterine Hemorrhage; Postmenopause. 


\section{INTRODUCCIÓN}

El sangrado uterino anormal en la posmenopausia es cualquier sangrado uterino que se presenta después de un año de amenorrea en la mujer en edad postmenopáusica, sin uso de terapia hormonal. Todas las mujeres posmenopáusicas con sangrado uterino deben ser evaluadas para descartar cáncer endometrial ya que esta enfermedad es la causa del sangrado en aproximadamente el 10 por ciento (1).

La hiperplasia endometrial se define como el engrosamiento del endometrio con proliferación de glándulas de tamaño y forma irregular y una mayor proporción entre glándulas y estroma. Representa un continuo de hallazgos histopatológicos. El sistema de Clasificación utilizado por la Organización Mundial de la Salud (OMS) y la International Society of Gynrcological Pathologists designa cuatro tipos distintos con potencial maligno variable (hiperplasia simple, hiperplasia compleja, hiperplasia simple atípica e hiperplasia compleja atípica) (2).

Las hiperplasias se clasifican como simples o complejas con base en la ausencia o presencia de anomalías arquitectónicas de las glándulas 1endometriales (OMS). Estas anomalías consisten en aglomeración y complejidad de las glándulas. Es muy importante señalar que la hiperplasia se clasifica además como atípica si muestra atipia nuclear de las células glandulares endometriales. Las hiperplasias endometriales atípicas tienen una relación clara con el desarrollo ulterior de adenocarcinoma (3).

Como factores de riesgo para padecer dicha entidad se encuentra la obesidad, es un problema de salud pública en el mundo occidental que se asocia a un incremento de hasta cinco veces mayor (4). En mujeres premenopáusicas, la obesidad y el síndrome de ovario poliquístico están asociados a ciclos anovulatorios, y como consecuencia de ello, el endometrio se encuentra expuesto a la acción de los estrógenos en ausencia de la acción reguladora de la progesterona.

En la posmenopausia, la obesidad conlleva a exposición del endometrio a cantidades elevadas de estrógenos provenientes de la trasformación periférica de la androstendiona (A4) a estrona (E1) y estradiol (E2), los cuales, en ausencia del efecto regulador de la progesterona, estimulan la proliferación endometrial que puede progresar a hiperplasia endometrial (5).

El sangrado uterino anormal es la forma como más comúnmente se manifiesta la hiperplasia endometrial (menstruación excesiva, menorragias, sangrado intermenstrual) aunque en algunos casos puede cursar de manera asintomática. Los síntomas más habituales de la hiperplasia son: Hemorragia vaginal entre períodos menstruales o después de la menopausia, sangrado menstrual abundante, períodos que duran más de lo normal, dolor durante las relaciones sexuales, amenorrea, períodos anovulatorios (6).

La ecografía transvaginal es el mejor método de evaluación en mujeres, tanto sintomáticas como asintomáticas, para el diagnóstico de patología endometrial (7). El endometrio se define como engrosado cuando según medidas ecográficas se encuentra, en mujeres premenopáusicas mayor a 8 $\mathrm{mm}$ en fase proliferativa, mayor a $14 \mathrm{~mm}$ en fase secretora; y en mujeres menopáusicas mayor a $5 \mathrm{~mm}$ (pacientes sin tratamiento de sustitución hormonal) (8).

La biopsia endometrial para el estudio histológico es la que determina el diagnóstico de la hiperplasia, la misma puede ser obtenida mediante el uso de cureta, aspiración o por histeroscopia (9). La hiperplasia endometrial es una patología trascendente en el ámbito de salud femenina ya que está ligado de manera significativa con cáncer en mujeres (10). La relación de la hiperplasia endometrial y el adenocarcinoma de endometrio ha quedado demostrada: la mayoría de los adenocarcinomas tienen hiperplasia de endometrio previas entre un 33 y $75 \%$ de los casos (11).

El propósito de este trabajo es determinar los hallazgos anatomopatológicos más prevalentes en pacientes que consultaron por sangrado uterino de la posmenopausia, encontrar los factores asociados más frecuentes y de esa manera encaminar a un protocolo de trabajo en nuestro servicio para prevenir y tratar de la manera más eficiente posible a las pacientes con hemorragia uterina de la posmenopausia.

\section{METODOLOGÍA}

Es un estudio observacional, descriptivo y temporalmente retrospectivo. El muestreo es no probabilístico de casos consecutivos. La población accesible son pacientes posmenopáusicas con sangrado uterino anormal que fueron sometidas a biopsia de endometrio en la cátedra de Ginecología y Obstetricia del Hospital de Clínicas de la Universidad Nacional de Asunción en el período enero 2019 a diciembre 2020, de las cuales se tomaron muestra que fueron enviadas al servicio de Anatomía Patológica del mismo hospital.

Se excluyó a pacientes que hayan tenido su último sangrado menstrual en un período menor a un año, con terapia de sustitución hormonal o que se han realizado histerectomía. Las variables incluidas son obstétricos y 
reproductivos, diagnóstico ecográfico, biopsia endometrial, hallazgo histopatológico, factores de riesgo, procedimiento quirúrgico.

Se respetarán los principios de respeto y autonomía manteniendo el anonimato de las pacientes seleccionadas para el estudio y manteniendo sus datos en la confidencialidad. Se respetará el principio de beneficencia ya que este estudio es de utilidad para ampliar el conocimiento tanto de las pacientes como del servicio, y de esta manera brindar una mejor atención. También se respetará el principio de justicia ya que no hay discriminaciones en la incorporación de sujetos para el estudio. Todas las pacientes seleccionadas tenían indicaciones clínicas para realizarse biopsia endometrial y ecografía ginecológica, la participación de las mismas no reportó gastos adicionales para ellas.

\section{RESULTADOS}

Se incluyó a 91 mujeres con edades comprendidas entre los 46 y 86 años con una media de 57,31 $\pm 8,69$ años y una mediana de 55 años lo que implica que al menos la mitad de las participantes es mayor a 55 años. La edad más frecuente fue 50 años. Sobre los antecedentes obstétricos el 68,1\% era multípara, el 5,5 $\%$ primípara y el $4,4 \%$ nulípara.

La cirugía más realizada fue histeroscopía diagnóstica en el $90,21 \%$ de los casos, en el 6,6 \% se realizó legrado biópsico fraccionado, el resto fueron otros procedimientos. El grosor del endometrio estuvo entre 1 y $36 \mathrm{~mm}$ con una media de $10,96 \pm 8,5 \mathrm{~mm}$ y una mediana de $8 \mathrm{~mm}$. Analizando el grosor del endometrio de forma cualitativa se observa que el grosor es normal (menos de $4 \mathrm{~mm}$ ) en $28,6 \%$ de las pacientes, está engrosado (más de $4 \mathrm{~mm}$ ) en $56 \%$ y muy engrosado (más de $20 \mathrm{~mm}$ ) en $15,4 \%$ de las pacientes.

En cuanto a los factores de riesgo, el más frecuente fue obesidad con $30,8 \%$, seguido por hipertensión con $24,2 \%$ y diabetes mellitus tipo 2 en el $11 \%$ de las pacientes. El 25,3\% no tiene factores de riesgo. Sobre el hallazgo histopatológico se puede ver que el $30,8 \%$ fue pólipo endometrial, el 24,2 \% mucosa endometrial normal, el $11 \%$ adenocarcinoma. Estos datos se ven en detalle en la tabla 1.

TABLA 1. DISTRIBUCIÓN DE LOS HALLAZGOS HISTOPATOLÓGICOS (N=91)

\begin{tabular}{lcc}
\hline \multicolumn{1}{c}{ Hallazgo } & Frecuencia & Porcentaje \\
\hline Pólipo endometrial & 28 & 30,8 \\
Mucosa endometrial normal & 22 & 24,2 \\
Adenocarcinoma & 10 & 11,0 \\
Sin datos & 9 & 9,9 \\
Hiperplasia endometrial sin atipas & 6 & 6,6 \\
Mioma submucoso & 4 & 4,4 \\
Carcinoma & 4 & 4,4 \\
Endometrio atrófico & 3 & 3,3 \\
Metaplasia escamosa sin atipias & 2 & 2,2 \\
Endometritis & 1 & 1,1 \\
Adenomiosis & 1 & 1,1 \\
Material insuficiente & 1 & 1,1 \\
\hline
\end{tabular}

Al buscar relación entre las medias de grosor endometrial y las alteraciones histopatológicas encontradas se puede ver que estas medias son

\section{DISCUSIÓN}

El sangrado uterino anormal ya sido investigado en el Hospital de Clínicas por Franco-Domínguez quien llega a la conclusión de que la prevalencia de hemorragia uterina anormal de causa orgánica es 16,3 \%. El principal motivo de consulta es la hipermenorrea $(63,3$ $\%$ ). Los diagnósticos finales fueron mioma uterino (57 \%) y las patologías endometriales. La mayoría fue sometida a cirugía (71 \%) y $38 \%$ presentó anemia (12). estadísticamente significativas al realizar un ANOVA $(p<0,001)$; aquellas con adenocarcinoma tienen una mayor media de grosor que los otros hallazgos.
La mayoría de las mujeres incluidas en la investigación son multíparas $(68,1 \%)$ lo que coincide con investigaciones similares con un 44,9\% (13), esto se debe en parte a las características de la muestra, puesto que son mujeres adultas, la mediana en este caso fue de 50 años, lo que indica que la mitad de las mujeres en la muestra tiene 50 años o más.

En cuanto al grosor está engrosado (más de $4 \mathrm{~mm}$ ) en $71,4 \%$ de los pacientes, en la investigación de SuárezEnriquez se reportó que la valoración por ultrasonido 
dividió las pacientes en 2 grupos de acuerdo al grosor endometrial (menos de $12 \mathrm{~mm}$ y más $13 \mathrm{~mm}$ ), con predominio de $83 \%$ en pacientes con endometrio mayor que $13 \mathrm{~mm}$ (14).

Este análisis de grosor se hizo por ecografía. La ecografía endovaginal (EVS) es el procedimiento de elección para la evaluación inicial de enfermedades benignas del tracto genital femenino. Cuando los hallazgos de la ecografía son indeterminados, a menudo se realiza una evaluación adicional con imágenes de resonancia magnética (MRI) debido a su excelente diferenciación de tejidos blandos, capacidades multiplanares y ausencia de radiación ionizante (15).

En cuanto a los factores de riesgo, el más frecuente fue obesidad con $30,8 \%$, seguido por hipertensión con $24,2 \%$ y diabetes mellitus tipo 2 en el $11 \%$ de las pacientes. En un estudio similar se encontró que la obesidad tiene una frecuencia de $47,5 \%$, la hipertensión 69,8 \% y la diabetes tipo 2 de 23,5 \% (16), como se puede ver estos datos son mayores a los encontrados en esta muestra, una explicación puede ser las características propias de la población estudiada.

Sobre el hallazgo histopatológico se puede ver que el $30,8 \%$ fue pólipo endometrial, el $24,2 \%$ mucosa endometrial normal, el $11 \%$ adenocarcinoma, en otra investigación el endometrio proliferativo fue el hallazgo histopatológico más común $(37,7 \%)$ seguido del endometrio secretor (31,2 \%) (17). En la investigación de Franco-Domínguez los hallazgos más encontrados fueron mioma y pólipo endometrial (12).

\section{CONTRIBUCIÓN DE LOS AUTORES}

JG: Concepción y diseño del estudio, procesamiento y análisis de los datos, redacción del manuscrito, revisión de la literatura, aprobación de la versión final del documento. NA: Concepción y diseño del estudio, procesamiento y análisis de los datos, revisión crítica del manuscrito, revisión de la literatura, aprobación de la versión final del manuscrito.

\section{REFERENCIAS}

1. Ayala-Peralta $F$, Rodríguez-Herrera $M$, Izaguirre-Lucano $H$, Ayala-Palomino R, Quiñonez-Vásquez L, Ayala-Moreno D, et al. Sangrado uterino anormal en la postmenopausia: diagnóstico y manejo. Rev Peru Investig Matern Perinat. 2016;5(1):56-64.

https://dx.doi.org/10.33421/inmp.201656

2. Hoffman BL. Williams ginecología: Tercera edición. 2017.

3. Pérez Manzano G, Restrepo Pulgarín JC. Cáncer de endometrio: una actualización en el diagnóstico y tratamiento; revisión de la literatura. [Barranquilla]: Universidad Libre Seccional Barranquilla; 2016.

4. Petridou E, Belechri M, Dessypris N, Koukoulomatis P,
Diakomanolis $\mathrm{E}$, Spanos $\mathrm{E}$, et al. Leptin and body mass index in relation to endometrial cancer risk. Ann Nutr Metab. 2002;46(3-4):147-51. https://doi.org/10.1159/000063081

5. Carreras R, Mancebo G. Obesidad y cáncer de endometrio. Med Clínica. 2007;128(5):176-7. https://doi.org/10.1016/S0025-7753(07)72528-9

6. Montgomery BE, Daum GS, Dunton CJ. Endometrial hyperplasia: a review. Obstet Gynecol Surv. 2004;59(5):368-78. https://doi.org/10.1097/00006254200405000-00025

7. Sepúlveda OAB, Rúa LJZ. Hiperplasia endometrial y criterios ecográficos: reporte de caso y revisión de la literatura. Med UPB. 2018;37(2):149-53.

https://doi.org/10.18566/medupb.v37n2.a09

8. Wolfman W, Leyland N, Heywood M, Singh SS, Rittenberg DA, Soucy R, et al. Asymptomatic endometrial thickening. J Obstet Gynaecol Can. 2010;32(10):990-999. https://doi.org/10.1016/s1701-2163(16)34690-4

9. Perera Boza OM, Molina Peñate L, Torres Toledo J. Caracterización de la hiperplasia endometrial en el Hospital Ginecobstétrico Docente "Profesor Eusebio Hernández". Rev Cuba Obstet Ginecol. 2014;40(3):307-18. URL.

10. Torriente Hernández B, Berty Pérez A, Martínez Camilo RV, Vasallo Pastor N. Valor de ultrasonido abdominal en el diagnóstico de afecciones endometriales en la mujer de mediana edad. Rev Cuba Obstet Ginecol. 2000;26(2):69-73. URL.

11. García Ayala E, Cárdenas Mastrascusa L, Sandoval Martínez D, Mayorga Anaya H. Hiperplasia endometrial: análisis de serie de casos diagnosticados en biopsia endometrial. Rev Chil Obstet Ginecol. 2010;75(3):146-52. https://dx.doi.org/10.4067/S0717-75262010000300002

12. Franco Domínguez E. Hemorragia uterina anormal orgánica. Rev Nac Itauguá. 2012;4(2):15-22. URL.

13. Romo Aguirre C, Colín Mejía DH. Correlación entre el diagnóstico clínico de un sangrado uterino anormal y el resultado anatomopatológico. Acta Médica Grupo Ángeles. 2020;18(1):7-10. URL.

14. Suárez Enríquez J, Pérez López J del C, Martínez Hernández CM. Concordancia entre histeroscopía e histopatología en pacientes con sangrado uterino anormal. Horiz Sanit. 2019;18(1):75-81. t https://doi.org/10.19136/hs.a18n1.2259

15. Kinkel K, Ascher SM, Reinhold C. Benign Disease of the Uterus. En: Hodler J, Kubik-Huch RA, von Schulthess GK, editores. Diseases of the Abdomen and Pelvis 2018-2021: Diagnostic Imaging - IDKD Book. Cham (CH): Springer; 2018.

16. Valenzuela-Islas $H A$, Frías-Mendívil $M$, Luis-Zárate $H$, Valenzuela-Islas HA, Frías-Mendívil $M$, Luis-Zárate $H$. Correlación entre hallazgos histeroscópicos y reportes histopatológicos en pacientes con sangrado uterino anormal. Ginecol Obstet México. 2017;85(11):748-54. https://doi.org/10.24245/gom.v85i10.1590

17. Chapagain S, Dangal G. Clinical and Histopathological Presentation of Abnormal Uterine Bleeding in Perimenopausal Women in Tertiary Center of Nepal. J Nepal Health Res Counc. 2020;18(2):248-52. t https://doi.org/10.33314/jnhrc.v18i2.2512 